\title{
Technical analysis of XML document in WEB
}

\author{
HU Jingfang1, a, LI Busheng1, b \\ 1 School of Information Engineering, Jingdezhen Ceramic Institute, \\ Jingdezhen, 333403, China \\ aemail: jdzhjf@163.com,bemail:abulbs@163.com
}

\begin{abstract}
In the Web page XML document that has a lot of technology, this paper introduces the XML data shown by CCS and XSL in two ways, and the advantages and disadvantages of these two kinds of display mode to do a comparison. This paper also to the XML document on the server and browser display methods are analyzed, and put forward the solving method.

Keywords: XML documents; CSS; XSL; display technology

\section{The basic way to display the XML document}

XML display a variety of technical solutions, including the use of CSS, XSL, JavaScript and XML Data Island [1]. In the application of Web to display XML data have two main options: create a cascading style sheet CSS(Cascading Style Sheets) to produce these data based on HTML format, or make Liu needle on XML technology extensible style sheet language XSL(Extensible style Language).
\end{abstract}

\subsection{CSS}

CSS in 1996 as the relevant style attribute information (such as font and border) standard method is added to the HTML document and put forward [2]. Between the HTML bear the CSS mark and HTML mark backwards compatible task. For a certain style mansion in specific XML elements, CSS is a very simple, easy to understand language. Any common word processing software with CSS supports most style. For example: select the font type, thickness, size, background color, spacing of various elements, elements of the border around the and so on. However, all is not in the document style information stored, but placed in a called style single independent document. Change the style sheet is visible in many different ways to format an XML document, suitable for different purposes - print, Web, display and other purposes. The work to be done is only the designated media style, does not need to change the content of the document.

\section{$1.2 \mathrm{XSL}$}

XSL is for XML examples: a markup language defined, it provides powerful 
functions than CSS, such as the elements and then sorting, filtering mail. XSL can make the Web browser directly according to the different needs of users of the representation of the butterfly change document (such as data show the sequence of changes), which needs no month server interactive communication. Through the transformation of the style sheet, the same document can provide different styles.

\section{3 comparisons of CSS and XSL}

CSS and XSL are repeated to some extent. CSS compatible with the browser, more widely than XSL. The function of XSL is more than CSS, but its strongman, complexity of influence. CSS only allows formatting element content is not allowed to change to re arrange the content, must be based on the content or attribute element as the element selection format in different ways such as a signature or add text like simple, extra. XSL is very suitable in XML document contains only the minimum data, and once the data around without HTML decorative case. XSL will eventually form a visible standard. The use of XSL to isolate key data from the page, such as the masthead, guide bar and a signature etc.. The use of CSS to the data document contains all the items. XML ten XSL allows data document and Web page document separation exist alone, so that the XML ten XSL documents easier maintenance and treatment.

\section{Display method of XML}

Display XML data document in Web, follow the following steps:

(1) The formation of the XML data document legal;

(2) Using the display form of CSS or XSL design of XML document;

(3) In the Browser or Server end of the XML document and the display style file binding, generate browse page;

(4) Browsing the data in Browser.

As can be seen, to display XML data document, must define the display form. The main use of CSS or XSL language. Using the style of CSS or XSL display definition of XML data document, all exist in display or display method of Browser terminal display problem in Server terminal [3].

CSS style sheets in different places, the scope has the function of different. The style sheet is divided into inline style sheets and external style sheets see body placed in the introduction page, external, external import in three ways [4].

(1) Inline style sheets, is actually the intermediate style sheet rules on the $<$ HEAD $>$ and $<$ /HEAD $>$, so that the style sheet to the whole of the current HTML page effect. The specific method for direct insertion method, which is a sign of individually specified HMTL page, the provisions of its style. Such as writing: <Table style = "font-size: 10pt; color: blue "> this statement defines the form of character size is 10pt, the color of blue.

(2) External style sheet. Through the introduction of an external style sheet, 
realize the style definitions. There are mainly two kinds of methods:

The use of $<$ LINK $>$ markers linked by an external style sheet, which specifies that the style definition file play. The basic grammatical forms such as:

$<$ LINK REL = STYLESHEET HREF = "example. cSs" >

The provision of this statement is defined using the HTML document and on the same day recorded in example.css style. An HTML document can refer to multiple external style sheets, for example:

$<$ LINK REL $=$ STYLESHEET HREF $=$ "example.css" $>$

$<$ LINK REL = STYLESHEET HREF = "style/other: css" >

First of all links of the example.css document as the default style sheet when the style definitions produce punching clamp, should first meet the former.

The use of Import import style sheet information, which is external style sheet information into the page. Import command to an external style sheet information into the page, exist in the $<$ STYLE $>$ and $</$ STYLE $>$ markers. For example:

$<$ STYLE TYPE = "text/css" >

Import "example.css";

Import "style/other. css";

$</$ STYLE $>$

Obviously, this method can also refer to multiple external style sheet information, the style sheet has the function of priority in order to set import. Display style defined by XSL, because not all browsers support XML and XSL, so there are display or in specific display method of Browser terminal display problem in Server terminal.

\subsection{Display method of Server terminal}

In the XML servers into a method of HTML XSL is seen in a browser XML documents into HTML, tips the method is to make JavaScript or VbScript script language to use an XML parser to convert. But when the browser does not support the XML parser, this method does not work. In order to make the XML data that is visible to all browsers, we must transform the XML document on the server, and send it to the browser as the pure HTML send. This method is becoming a major part of the Internet information server tasks in the future.

The method when used to make sure that the XML file: no reference to the XSL file, the XSL file is not a reference to the XML file, and attention to a server XML file can choose many different XSL file conversion. At the end of the server will be converted into XML HTML. The following is seen to convert XML files VbScript needs a simple source code on the server.

book catalog. asp:

$<\%$ "Load the XML

Set xml=Server.CreateObject("Microsoft. XMLDOM")

$\mathrm{XML}$. async $=$ false

xml. 1oad(ServerlMapPath("bus. xml”))

'Load the XSL

Set xsl=ServerCreateobject("Microsoft. XMLDOM") 
XSL. async $=$ false

XSL. 1oad(ServerMapPath("bus. xsl”)),

Transform the fi1e

Response. Write(xml. transformNode(xsl))\%>

In the code, first create the Microsoft XML parser (XMLDOM) an instantiation, and load a XML file into memory; and then created another instantiation of the parser, and the XSL document is loaded into memory; finally using XSL document conversion XML document, and the result is returned to the browser.

\subsection{Display method of Browser terminal}

If the browser supports XML, you can in the browser using XSL to convert documents into HTML. The common method is to add an XSL style sheet to the XML file, and then let the browser to convert. But this method in the XML file to include a style sheet reference, and invalid on browsers that do not support XSL. Another method is to use the JavaScript or VbScript script language to from the XML to HTML conversion. The script can be used, the browser test details, according to the browser and the requirements of users using different style sheets, support different browsers and different user needs.

The above two methods, also have to ensure that the XML file is not a reference to the XSL file, the XSL file is not a reference to the XML file. Another note is: a XML file can be used in many different XSL file conversion. Conversion to HTML in a browser XML, the following is on the client using JavaScript to convert XML files to HTML need the source code.

book_Catalog. html:

$<\mathrm{Html}><$ body $>$

$<$ script language="JavaScript" $>$

'Load XML

var xml=new ActiveXObject("Microsoft.XMLDOM")

$\mathrm{xml}$.async $=$ false

xml.load=("bus.xml")

'load the XSL

var xsl=new ActiveXObject("Microsoft.XMLDOM")

xsl.async $=$ false

xsl.load("bus.xsl")

'Transform

document.write(xml.transfromNode(xsl))

$<$ script $>$

$</$ body $>$

$</$ xml $>$

In the code, first create the Microsoft XML parser (XMLDOM) an instantiation, and the XML document is loaded into memory; another instantiation and then create the parser, and the XSL document is loaded into memory; finally using XSL document conversion XML document, write the results to the HTML document. 


\section{Conclusions}

In this paper using the code has been on versions of IE5.5 and above, the effect is very good. Because the XML scalability, as well as data and performance isolation, XML canonized numerous software company and support, practice XML technology appraisal system is widely used in a network, completed the HTML cannot realize the sorting, browsing and other functions.

\section{References}

[1] Ran Guangyao Li Jun, research on the technology of VxWorks XML documents display, computer and digital engineering, 2010.2

[2] (U) Bill Evjen, XML senior programming, Tsinghua University press, 2009.3

[3] (U) Hunter, (U) Luft, (U) Foster et al., translated by Wu Wenguo, XML introduction to the classic, Tsinghua University press,2009.2

[4] Li Gang, crazy XML lectures, publishing house of electronics industry,2009.11

[5] Luo Yan. Research on technology of heterogeneous exchange based on XML: (Master thesis). Nanchang: Nanchang University, 2011

[6] Chu Jiu Liang ed., Web front-end development technologies: HTML\CSS JavaScript, Tsinghua University press, 2013

[7] Tan Hanhua.XML language and built on the base of the XML three layer C/S model application. Coastal enterprises and technology.2008.5

[8] 